\title{
Robust PID Steering Control in Parameter Space for Highly Automated Driving
}

\author{
Mümin Tolga Emirler, ${ }^{1,2}$ İsmail Meriç Can Uygan, \\ Bilin Aksun Güvenç, ${ }^{2,4}$ and Levent Güvenç, \\ ${ }^{1}$ Department of Mechanical Engineering, İstanbul Technical University, 34437 İstanbul, Turkey \\ ${ }^{2}$ Mekar Labs, İstanbul Okan University, 34959 İstanbul, Turkey \\ ${ }^{3}$ Department of Control Engineering, İstanbul Technical University, 34469 İstanbul, Turkey \\ ${ }^{4}$ Department of Mechanical Engineering, İstanbul Okan University, 34959 İstanbul, Turkey
}

Correspondence should be addressed to Levent Güvenç; levent.guvenc@okan.edu.tr

Received 24 September 2013; Revised 27 November 2013; Accepted 29 November 2013; Published 4 February 2014

Academic Editor: Luis M. Bergasa

Copyright (C) 2014 Mümin Tolga Emirler et al. This is an open access article distributed under the Creative Commons Attribution License, which permits unrestricted use, distribution, and reproduction in any medium, provided the original work is properly cited.

\begin{abstract}
This paper is on the design of a parameter space based robust PID steering controller. This controller is used for automated steering in automated path following of a midsized sedan. Linear and nonlinear models of this midsized sedan are presented in the paper. Experimental results are used to validate the longitudinal and lateral dynamic models of this vehicle. This paper is on automated steering control and concentrates on the lateral direction of motion. The linear model is used to design a PID steering controller in parameter space that satisfies $D$-stability. The PID steering controller that is designed is used in a simulation study to illustrate the effectiveness of the proposed method. Simulation results for a circular trajectory and for a curved trajectory are presented and discussed in detail. This study is part of a larger research effort aimed at implementing highly automated driving in a midsized sedan.
\end{abstract}

\section{Introduction}

In recent years, intelligent vehicle systems and highly automated driving technologies have drawn interest among researchers. Many research efforts including, for example, the work reported in [1-3] have concentrated on semiautonomous and fully autonomous vehicles. Autonomous driving requires coordinated automation of the longitudinal and the lateral driving tasks of speed control and steering control, respectively. Desired path tracking of an autonomous vehicle requires the proper design and implementation of steering and speed controllers at the lower control level. This paper concentrates on automated steering control.

The basic automatic steering control algorithms found in the literature are based on PID (proportional-integralderivative) type controllers $[4,5]$. In these designs, the lateral deviation of the vehicle at a preview distance is fed back for controlling the vehicle's lateral dynamics. In [6], a robust PIDD controller is designed for automatic bus steering control as a solution of a benchmark problem. The yaw rate is measured in addition to lateral deviation measurements and is fed back for improving the control system performance. For the same benchmark problem, a discrete time add-on disturbance observer design is realized in [7]. Using the addon disturbance observer, the performance improvement is achieved without the need for yaw rate feedback. Another approach to automatic steering controller is to design nested PI and PID controllers [8]. A PI steering controller that reduces yaw rate tracking error is used to improve the vehicle steering dynamics and a PID controller is employed to reject the lateral deviation from the desired path due to road curvature disturbances [8].

In this paper, the parameter space approach based PID controller design is applied to automatic steering control. The theoretical background about the parameter space approach and an example of road vehicle yaw stability control can be found in the references [9-11]. The parameters exhibiting the 
largest variation in automatic steering control are the vehicle mass, the vehicle speed, and the road friction coefficient. In this paper, the controller parameter space for the two free coefficients of a PID controller which are chosen as the proportional gain $k_{p}$ and the derivative gain $k_{d}$ is obtained considering $D$-stability requirements. An overall solution region is calculated by intersecting the solution regions for exemplary points chosen from the boundary of the uncertain range of parameters. Robust PID coefficients satisfying $D$ stability are chosen from the overall calculated parameter space regions. The designed controller is applied to an experimentally validated nonlinear simulation model of a sedan used in autonomous vehicle tests by the authors.

The organisation of the rest of this paper is as follows. In Section 2, the linear vehicle model used in controller design and the experimental vehicle that it is based on are described. The nonlinear model of this experimental vehicle and model validation results are also presented in Section 2. In Section 3, the robust PID controller design is performed based on mapping $D$-stability boundaries into the parameter space. The simulation results in Section 4 illustrate the effectiveness of the designed controller. The paper ends with conclusions and recommendations for future work in Section 5.

\section{Vehicle Models and Experimental Vehicle}

2.1. Vehicle Steering Model. The vehicle steering behavior is modeled as single track model that also includes the dynamics of following the reference path as illustrated in Figure 1. The linear vehicle steering model is described in state-space form as

$$
\left[\begin{array}{c}
\dot{\beta} \\
\dot{r} \\
\Delta \dot{\psi} \\
\dot{y}
\end{array}\right]=\left[\begin{array}{cccc}
a_{11} & a_{12} & 0 & 0 \\
a_{21} & a_{22} & 0 & 0 \\
0 & 1 & 0 & 0 \\
V & l_{s} & V & 0
\end{array}\right]\left[\begin{array}{c}
\beta \\
r \\
\Delta \psi \\
y
\end{array}\right]+\left[\begin{array}{cc}
b_{11} & 0 \\
b_{21} & 0 \\
0 & -V \\
0 & 0
\end{array}\right]\left[\begin{array}{c}
\delta_{f} \\
\rho_{\mathrm{ref}}
\end{array}\right],
$$

where $\beta, r, V, \Delta \Psi, l_{s}$, and $y$ are vehicle side slip angle, vehicle yaw rate, vehicle velocity, yaw angle relative to the desired path's tangent, the preview distance, and lateral deviation from the desired path at the preview distance, respectively. The control input is the steering angle $\delta_{f} \cdot \rho_{\text {ref }}=1 / R$ is the road curvature where $R$ is the road radius. The remaining terms are

$$
\begin{aligned}
a_{11}=\frac{-\left(c_{r}+c_{f}\right)}{\widetilde{m} V}, & a_{12}=\frac{-1+\left(c_{r} l_{r}-c_{f} l_{f}\right)}{\widetilde{m} V^{2}}, \\
a_{21}=\frac{\left(c_{r} l_{r}-c_{f} l_{f}\right)}{\widetilde{J}}, & a_{22}=\frac{-\left(c_{r} l_{r}^{2}+c_{f} l_{f}^{2}\right)}{\widetilde{J} V^{2}}, \\
b_{11}=\frac{c_{f}}{\widetilde{m} V}, & b_{12}=\frac{c_{f} l_{f}}{\widetilde{J}},
\end{aligned}
$$

where $\widetilde{m}=m / \mu$ is the virtual mass, $\widetilde{J}=J / \mu$ is the virtual moment of inertia, $\mu$ is the road friction coefficient, $m$ is the vehicle mass, $J$ is the moment of inertia, $c_{f}$ and $c_{r}$ are the cornering stiffnesses, $l_{f}$ is the distance from the center

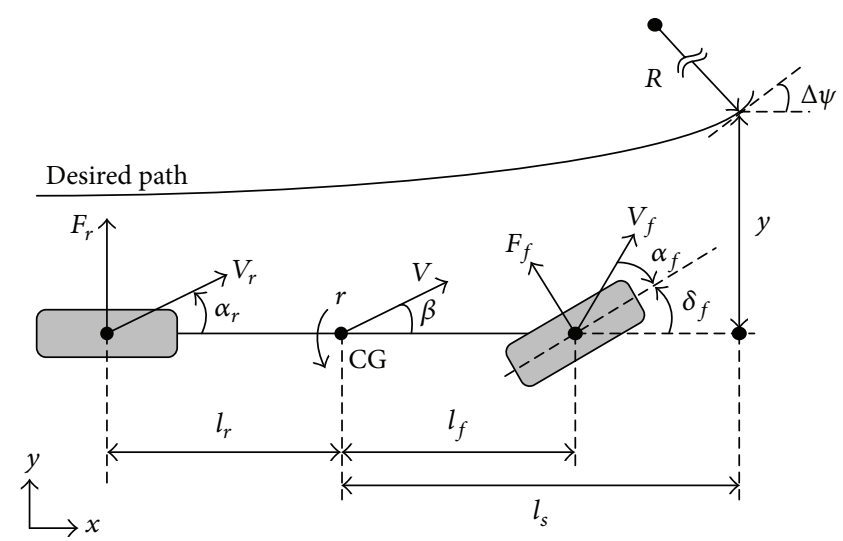

FIGURE 1: Vehicle steering model.

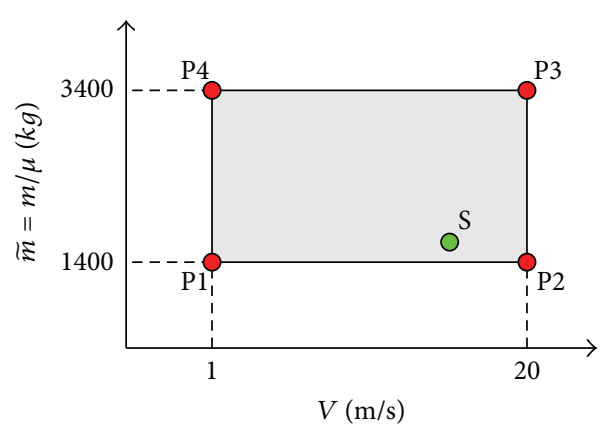

FIGURE 2: Uncertainty box.

of gravity of the vehicle (CG) to the front axle, and $l_{r}$ is the distance from the CG to the rear axle [7]. The values of the parameters used in this paper are $J=2392 \mathrm{kgm}^{2}, l_{f}=$ $1.07 \mathrm{~m}, l_{r}=1.53 \mathrm{~m}, l_{s}=2 \mathrm{~m}, c_{f}=72463 \mathrm{~N} / \mathrm{rad}$, and $c_{r}=$ $92492 \mathrm{~N} / \mathrm{rad}$. The vehicle mass, the vehicle velocity, and the road friction coefficient are taken as uncertain parameters within the ranges of $m \in[1400,1700]$ (kg) (the nominal value of mass is $1550 \mathrm{~kg}), \mu \in[0.5,1]$, and $V \in[1,20](\mathrm{m} / \mathrm{s})$, respectively. The virtual mass, then, is within the range $\widetilde{m}=$ $m / \mu \in[1400,3400](\mathrm{kg})$. The corresponding uncertainty box of virtual mass and vehicle speed is illustrated in Figure 2.

2.2. Experimental Vehicle. The experimental vehicle is a Fiat Linea midsized sedan. Three Fiat Linea midsized sedans were used by the authors and their colleagues in lateral dynamics testing [12], semiautonomous driving in a platoon [2], and autonomous path following experiments [13], respectively. The authors, therefore, have considerable experimental experience with this vehicle. The current paper uses the third of these vehicles in [13]. The steering controller designed in this paper will be tested on that vehicle which is shown in Figure 3. This is the drive-by-wire vehicle of İstanbul Okan University named Okanom [14]. Throttle, brake, and steering actuation signals are provided by a dSpace microautobox general purpose electronic control unit which is also used for all the low level computations. Available signals on the vehicle CAN bus are read by this microautobox. A personal computer operating under Linux is used as an upper level 


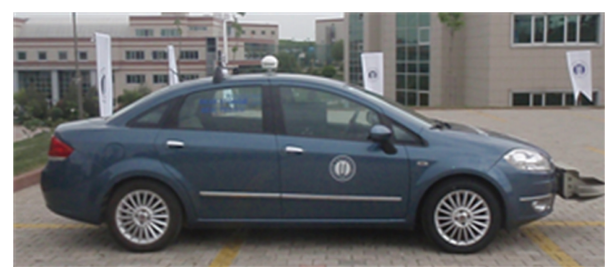

FIgURE 3: Experimental vehicle.

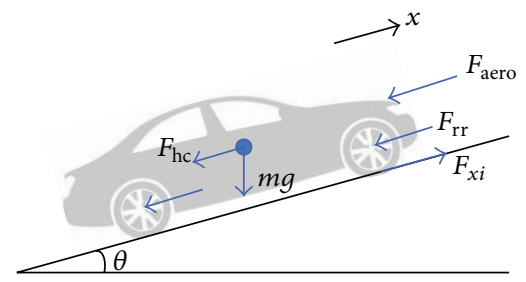

FIGURE 4: The resistive forces acting on the longitudinal dynamics of the vehicle.

control system. This PC collects data from the GPS receiver, the IMU unit, the Lidar in front of the vehicle, and the IEEE 802.11p vehicle to vehicle (V2V) communication modem and communicates with the low level microautobox controller. The GPS and the IMU signals are used in GPS/INS integration [15]. The GPS position of the vehicle is periodically sent by the V2V system to a nearby road side unit (RSU). This information is used to track the vehicle position on a remote computer and on smartphones. The path to be followed is recorded as a list of successive GPS waypoints which form the desired path in Figure 1.

2.3. Nonlinear Vehicle Model. The equations of motion for the longitudinal and the lateral dynamics of the nonlinear vehicle model are

$$
\begin{aligned}
m\left(a_{x}-r V_{y}\right)= & \sum_{i=f, r} F_{x i} \cos \delta_{i}-F_{y i} \sin \delta_{i} \\
& -\left(F_{\text {aero }}+F_{r r}+F_{h c}\right), \\
m\left(a_{y}+r V_{x}\right)= & \sum_{i=f, r} F_{x i} \sin \delta_{i}+F_{y i} \cos \delta_{i}
\end{aligned}
$$

while the equation of motion around the yaw axis is

$$
\begin{aligned}
I_{z} \dot{r}= & l_{f} F_{y f} \cos \delta_{f}-l_{r} F_{y r} \cos \delta_{r}+l_{f} F_{x f} \sin \delta_{f} \\
& -l_{r} F_{x r} \sin \delta_{r},
\end{aligned}
$$

where $F_{x i}$ and $F_{y i}$ are the longitudinal and the lateral tire forces. $f$ and $r$ represent the front and rear tires. $a_{x}, a_{y}, V_{x}, V_{y}$, and $I_{z}$ are the longitudinal acceleration at the CG, the lateral acceleration at the CG, the longitudinal velocity at the CG, the lateral velocity at the CG, and the moment of inertia about the yaw axis, respectively. Note that for the front wheel steered vehicle considered in this paper, the rear wheel steering angle $\delta_{r}=0$.

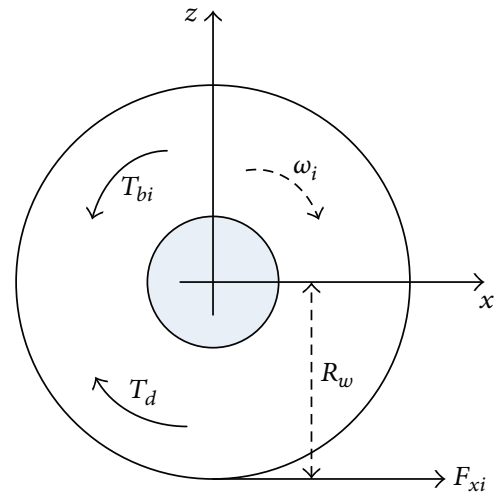

FIgURE 5: The forces and the torques acting on the wheel.

The resistive forces which affect the longitudinal dynamics of the vehicle are shown in Figure 4. The aerodynamic drag force $F_{\text {aero }}$ is given by

$$
F_{\text {aero }}=\frac{1}{2} A \rho C_{d} V^{2},
$$

where $A$ is the effective frontal area of the vehicle, $\rho$ is the mass density of air, $C_{d}$ is the drag coefficient, and $V$ is the velocity of the vehicle. The rolling resistance force $F_{r r}$ is determined as

$$
F_{r r}=C_{r r} m g \cos (\theta) \text {, }
$$

where $C_{r r}$ is the rolling resistance coefficient and $\theta$ is the road inclination angle. The gravitational slope resistance force $F_{h c}$ is modeled as

$$
F_{h c}=m g \cos (\theta) \text {. }
$$

The internal combustion engine (ICE) is modeled using a static engine map that defines the relationship between the inputs of throttle position $\alpha$, the engine speed $\omega$, and the output engine torque $T_{\text {ICE }}(\omega, \alpha)$. The engine torque output is transmitted to the wheels through the driveline as torque $T_{d}$ according to

$$
T_{d}=\eta_{t} i_{t} T_{\text {ice }}(\omega, \alpha)
$$

where $\eta_{t}$ is a static efficiency factor used to model mechanical losses and $i_{t}$ is the transmission ratio. These parameters are used to model the transmission of the vehicle.

The forces and torques acting on the wheel are shown in Figure 5. The moment balance at the center of the wheel is given by

$$
I_{w} \dot{\omega}_{i}=T_{d}-T_{b i}-F_{x i} R_{w}
$$

where $I_{w}$ is the moment of inertia of the wheel, $\omega$ is the angular velocity of the $i$ th wheel, $T_{b}$ is the braking torque on the $i$ th wheel applied through the brake system, $F_{x i}$ is the longitudinal tire force of the $i$ th wheel, and $R_{w}$ is the effective wheel radius. 

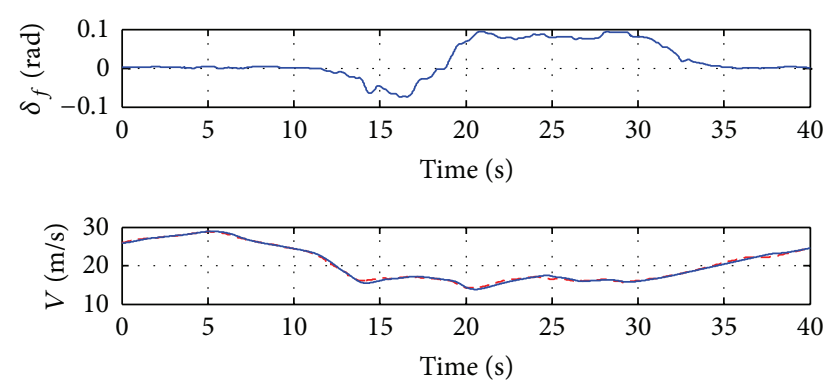

- - - Experimental result

- Simulation result

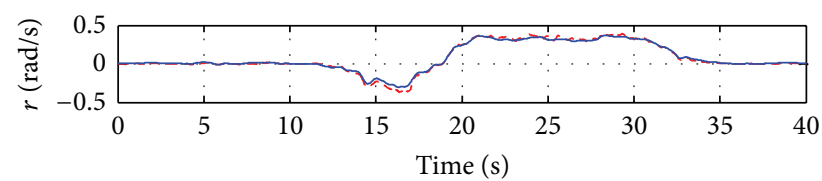

- - - Experimental result

- Simulation result

FIGURE 6: Comparison of test data and nonlinear vehicle simulation results for lateral dynamics.

The longitudinal velocities of the front and rear wheels can be determined as follows:

$$
\begin{aligned}
& V_{f x}=\sqrt{V_{x}^{2}+\left(V_{y}+l_{f} r\right)^{2}} \cos \alpha_{f}, \\
& V_{r x}=\sqrt{V_{x}^{2}+\left(V_{y}-l_{r} r\right)^{2}} \cos \alpha_{r},
\end{aligned}
$$

where the tire slip angles are

$$
\begin{gathered}
\alpha_{f}=\delta_{f}-a \tan \left(\tan \beta+\frac{l_{f} r}{V_{x}}\right), \\
\alpha_{r}=\delta_{r}-a \tan \left(\tan \beta-\frac{l_{r} r}{V_{x}}\right) .
\end{gathered}
$$

The longitudinal wheel slip ratio is defined as

$$
s_{i}= \begin{cases}\frac{R_{w} \omega_{i}-V_{i x}}{V_{i x}}, & R_{w} \omega_{i}<V_{i x} \text { (braking) } \\ \frac{R_{w} \omega_{i}-V_{i x}}{R_{w} \omega_{i}}, & R_{w} \omega_{i}>V_{i x} \text { (traction) },(i=f, r) .\end{cases}
$$

The Dugoff tire model is used for the calculations of the tire forces as

$$
\begin{aligned}
& F_{x i}=f_{i} C_{x i} s_{i}, \\
& F_{y i}=f_{i} C_{y i} \alpha_{i},
\end{aligned}
$$

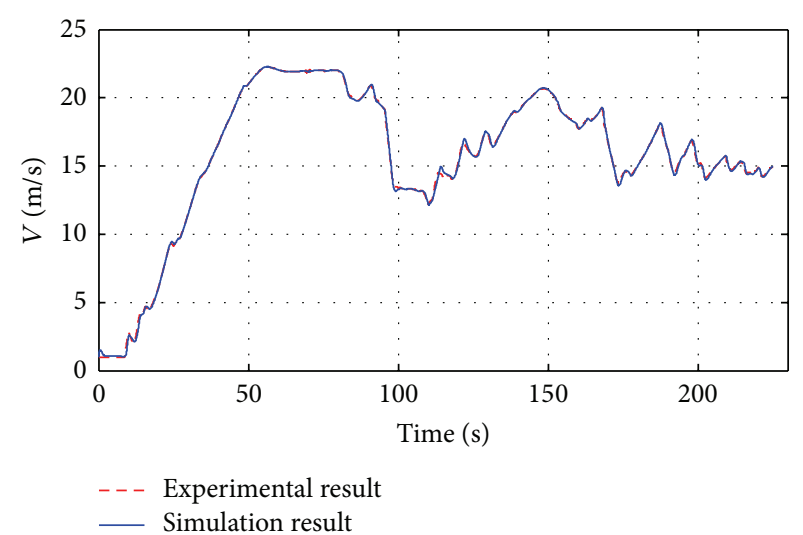

FIGURE 7: Comparison of test data and nonlinear vehicle simulation results for longitudinal dynamics.

where $C_{x i}$ and $C_{y i}$ are the longitudinal stiffness and the lateral cornering stiffness of the $i$ th wheel. The coefficients $f_{i}$ are determined using [2]

$$
\begin{gathered}
f_{i}= \begin{cases}1, & F_{R i} \leq \frac{\mu F_{z i}}{2} \\
\left(2-\frac{\mu F_{z i}}{2 F_{R i}}\right) \frac{\mu F_{z i}}{2 F_{R i}}, & F_{R i}>\frac{\mu F_{z i}}{2}\end{cases} \\
F_{R i}=\sqrt{\left(C_{x i} s_{i}\right)^{2}+\left(C_{y i} \alpha_{i}\right)^{2}} .
\end{gathered}
$$

Model validation studies were performed using the data obtained from the experimental vehicle test runs. The measured steering wheel and vehicle velocity were used as inputs to the nonlinear vehicle model. The simulated outputs for vehicle velocity and yaw rate were compared with the obtained experimental data. A comparison result from a test run is shown in Figure 6. A J-turn-like maneuver was applied to the vehicle. The nonlinear vehicle model results of the vehicle velocity and yaw rate were consistent with the obtained data from the experimental vehicle. In the test run shown in Figure 7, the experimental vehicle followed a velocity profile from the Grand Cooperative Driving Challenge [2]. The steering wheel input was zero. It can be seen from Figure 7 that the velocity obtained from the nonlinear vehicle model coincides with the experimental test result closely.

\section{Robust PID Steering Controller Design Using the Parameter Space Approach}

3.1. Mapping D-Stability Requirements into the Parameter Space. Similar to the approach in [11], D-stability requirements can be mapped into the parameter space.

Consider the plant given by

$$
G(s)=\frac{N(s)}{D(s)},
$$

where $N(s)$ represents the numerator of the plant and $D(s)$ represents its denominator. The real and imaginary parts of 


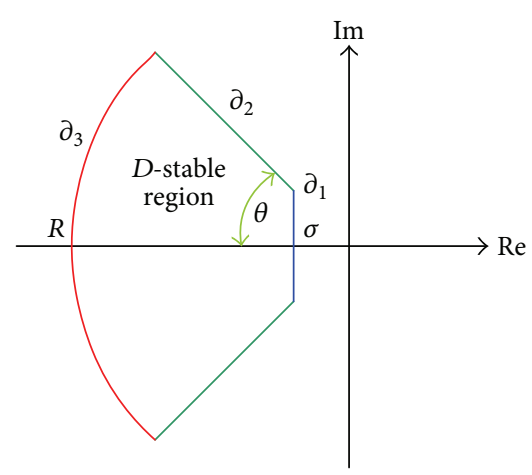

FIgURE 8: $D$-stable region in the complex plane.

the numerator and denominator can be defined as $N(j \omega)=$ $N_{R}(\omega)+j N_{I}(\omega)$ and $D(j \omega)=D_{R}(\omega)+j D_{I}(\omega)$.

The PID controlled closed loop system characteristic equation can be written as

$$
\begin{aligned}
p_{c}(s) & =s D(s)+\left(k_{p} s+k_{i}+k_{d} s^{2}\right) N(s) \\
& =a_{n+1} s^{n+1}+a_{n} s^{n}+\cdots+a_{1} s+a_{0}=0,
\end{aligned}
$$

where $n$ is the degree of the plant $G(s)$.

The Hurwitz stability boundary crossed by a pair of complex conjugate roots is characterized by the following equations:

$$
\begin{gathered}
\operatorname{Re}\left[p_{c}(j \omega)\right]=0, \\
\operatorname{Im}\left[p_{c}(j \omega)\right]=0, \quad \forall \omega \in(0, \infty] .
\end{gathered}
$$

This is called the complex root boundary (CRB).

There may be a real root boundary such that a single real root crosses the boundary at frequency $\omega=0$ as characterized by

$$
p_{c}(0)=0 \quad \text { or } \quad a_{0}=0 .
$$

This is called the real root boundary (RRB).

There may exist an infinite root boundary (IRB) which is characterized by a degree drop in the characteristic polynomial at $\omega=\infty$. This degree drop in the characteristic polynomial is characterized as

$$
a_{n+1}=0 .
$$

CRB, RRB, and IRB solutions parameterized by frequency $\omega$ can be plotted in the parameter plane of two free design parameters to show the Hurwitz stability regions of the given closed loop system. The free parameter pairs which provide Hurwitz stability can be chosen visually from the stable region of the parameter plane.

The aforementioned parameter space computation method to determine Hurwitz stability regions can be extended to specify relative stability regions such as $D$ stability. A closed loop system is $D$-stable when the roots of the closed loop characteristic equation lie in the $D$-stable region in the complex plane as depicted in Figure 8.

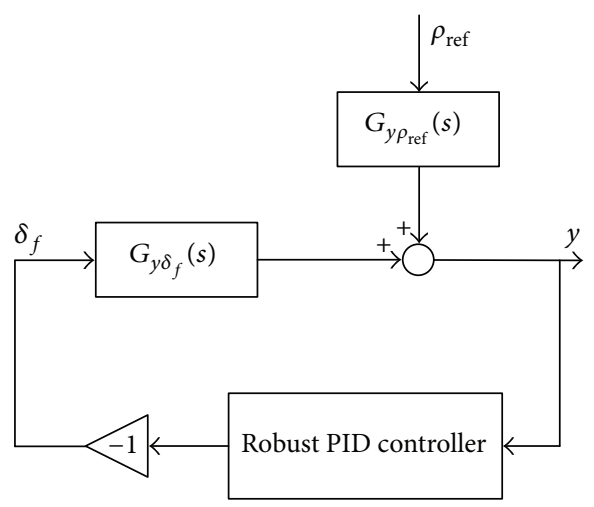

FIGURE 9: Control system structure.

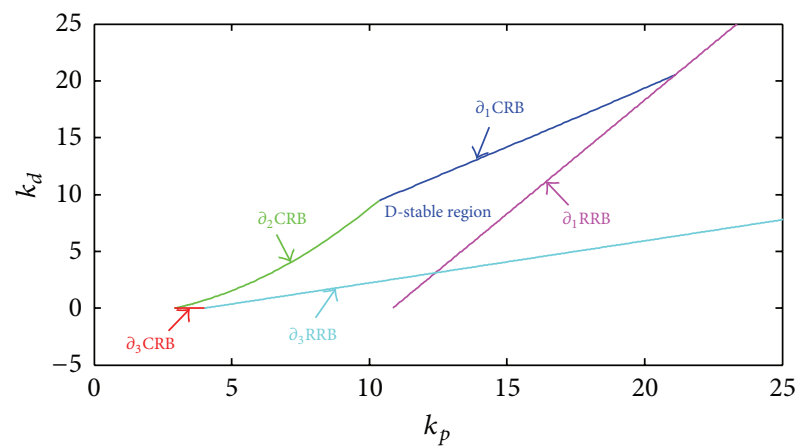

Figure 10: Detailed view of $D$-stability in parameter space for P1.

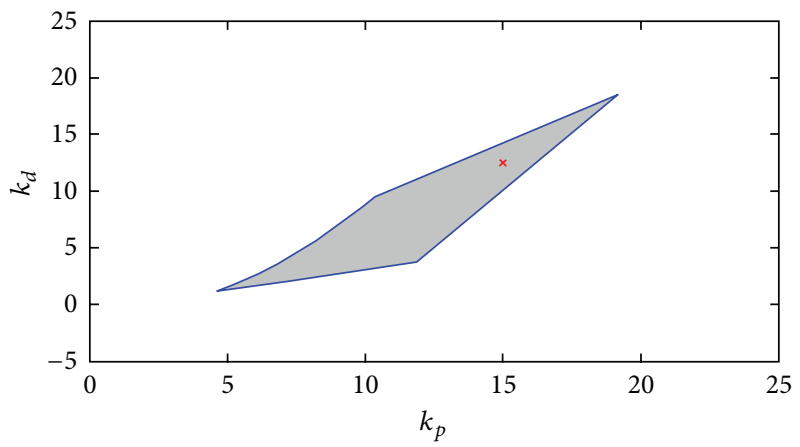

FIGURE 11: Overall $D$-stability solution region.

The boundary $\partial_{1}$ in Figure 8 can be mapped into the parameter space by using $s-\sigma$ instead of $s$ in (17) in order to shift the stability boundary to $\partial_{1}$ in the complex plane. Solving for two free parameters in (18) for CRB and (19) for $\mathrm{RRB}$ and then plotting results will result in the $\partial_{1}$ boundary in the parameter space. For the $\partial_{1}$ boundary, there is no IRB because $s$ is never equal to infinity in the $D$-shaped region. For mapping the $\partial_{2}$ boundary, use $r e^{j \theta}$ for $s$ with constant $\theta$ in (17) and parameterize $r$ in (18) to obtain the CRB of $\partial_{2}$. No RRB and IRB solution exists because $r$ is never equal to zero or infinity. Lastly, the $\partial_{3}$ boundary maps into the parameter space by substituting $s$ with $R e^{j \theta}$ where $R$ is constant and the map is parameterized over $\theta$ in (17). This results in CRB for changing $\theta$ and RRB for $\theta=0^{\circ}$. 


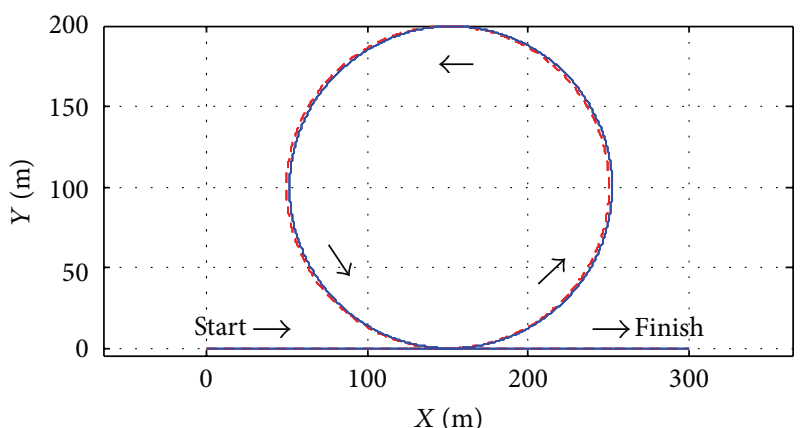

- - Desired

- Actual

(a)

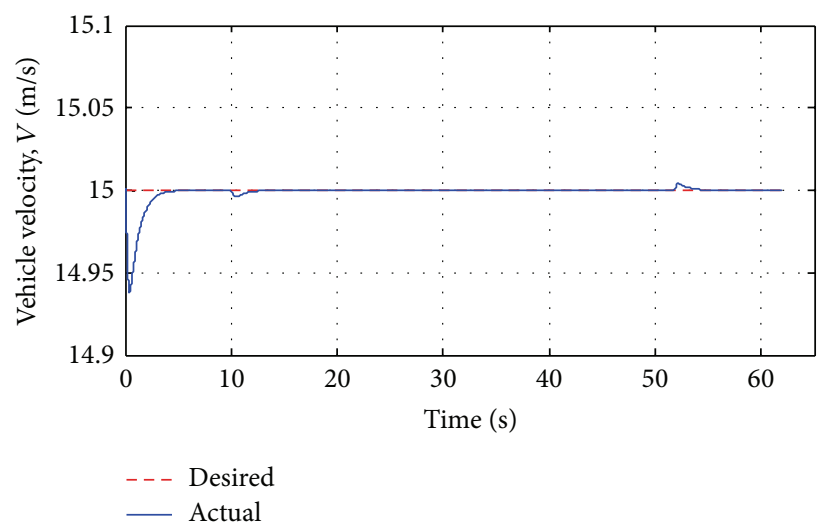

(c)

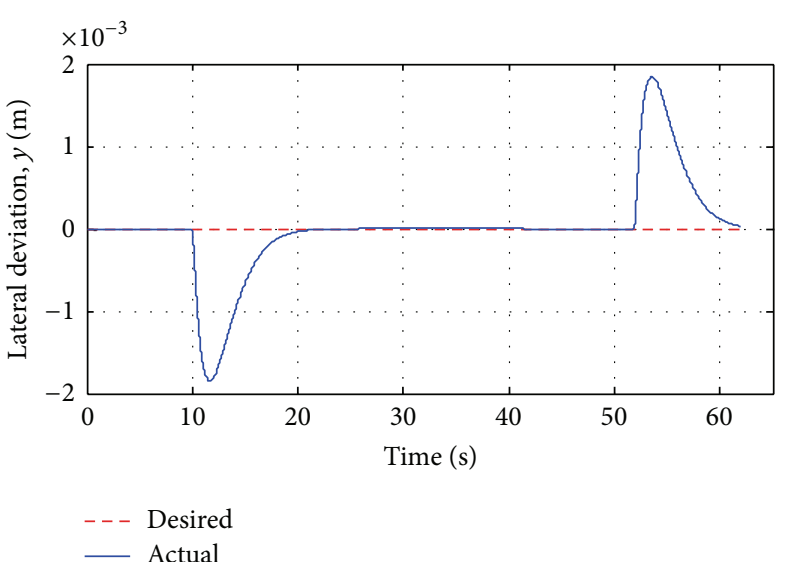

(b)

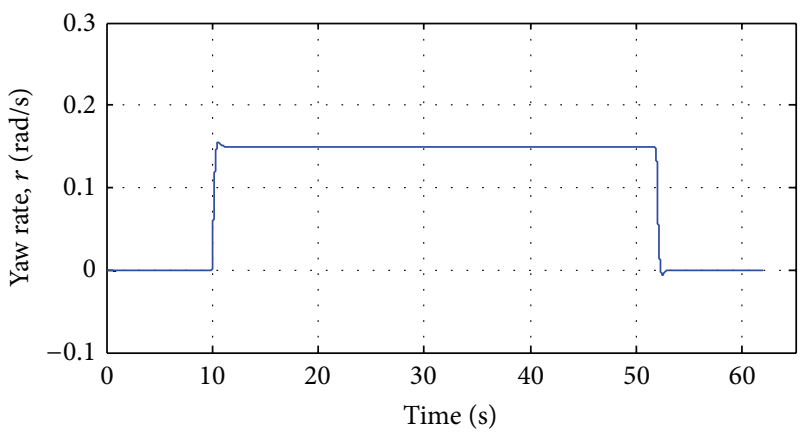

FIGURE 12: Simulation results for circular trajectory.

3.2. Application to Automatic Steering Control. The vehicle steering dynamics state-space model corresponding to (1) can be expressed in standard form as

$$
\dot{x}=A x+B u .
$$

Using the state-space form, the transfer function $G_{y \delta f}$ from the steering angle $\delta_{f}$ to the lateral deviation $y$ is written as

$$
G_{y \delta_{f}}=\left[\begin{array}{llll}
0 & 0 & 0 & 1
\end{array}\right](s I-A)^{-1}\left[\begin{array}{c}
b_{11} \\
b_{21} \\
0 \\
0
\end{array}\right]
$$

and the transfer function from the road curvature $\rho_{\text {ref }}$ to the lateral deviation $y$ is described as

$$
G_{y \rho_{\mathrm{ref}}}=\left[\begin{array}{llll}
0 & 0 & 0 & 1
\end{array}\right](s I-A)^{-1}\left[\begin{array}{c}
0 \\
0 \\
-V \\
0
\end{array}\right] .
$$

These transfer functions are used in designing the robust PID controller for the automatic steering system. The control system structure is illustrated in Figure 9.
The robust PID controller is designed based on the parameter space approach. The $D$-stability requirements are taken into consideration. The $D$-stability boundaries (shown in Figure 8) are formed by assuming roots no closer than 0.5 to the imaginary axis and no further in magnitude than 2.7 from the imaginary axis ( $\sigma=0.5$ and $R=2.7$ ). A minimum damping ratio corresponding to $\theta=45^{\circ}$ is determined as 0.707 .

Two parameters of the PID controller are selected as free design parameters. In this paper, these free parameters are chosen as the proportional gain $k_{p}$ and the derivative gain $k_{d}$ of the PID controller. The integral gain $k_{i}$ of the PID controller is determined as a fixed parameter by the designer. Here, $k_{i}$ is selected as 5 .

Figure 10 shows the solution region for the P1 vertex of the uncertainty box shown in Figure 2. $\partial_{1}$ CRB and RRB, $\partial_{2} \mathrm{CRB}$, and $\partial_{3} \mathrm{CRB}$ and $\mathrm{RRB}$ of the $D$-stability bounds are depicted with different colors. The intersection of these bounds determines the boundary of the $D$-stable region in the $k_{p}-k_{d}$ plane.

The overall solution region which combines all the solutions for the vertices of the uncertainty box in Figure 2 is shown in Figure 11. The design point for $k_{p}$ and $k_{d}$ is selected 


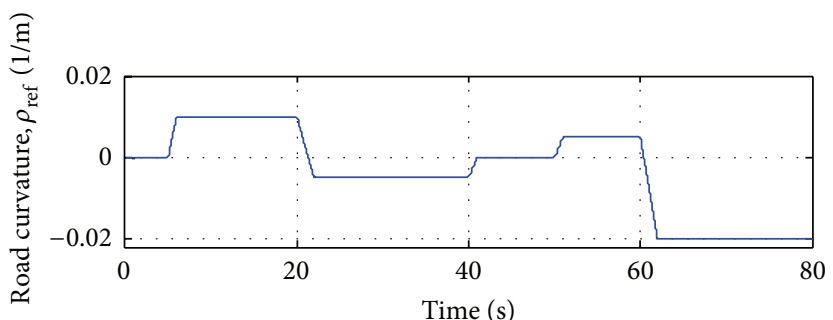

(a)

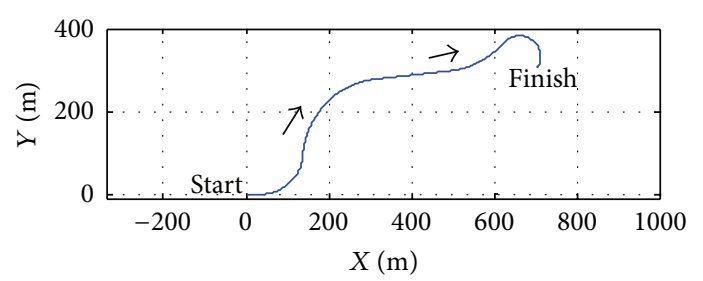

(c)

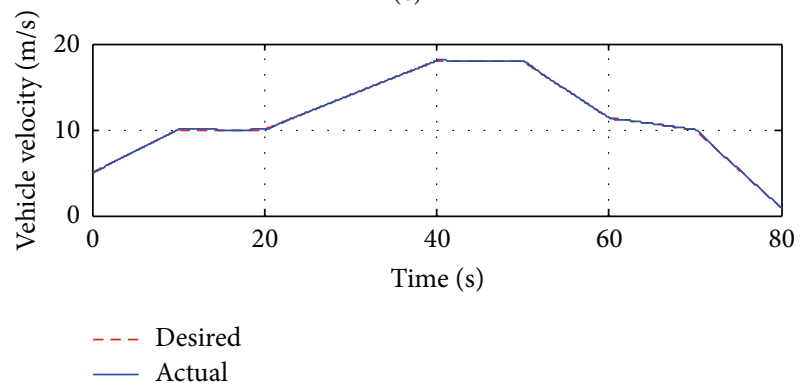

(e)

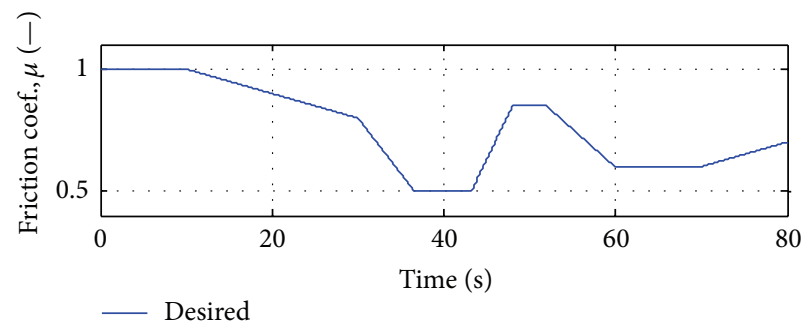

(b)

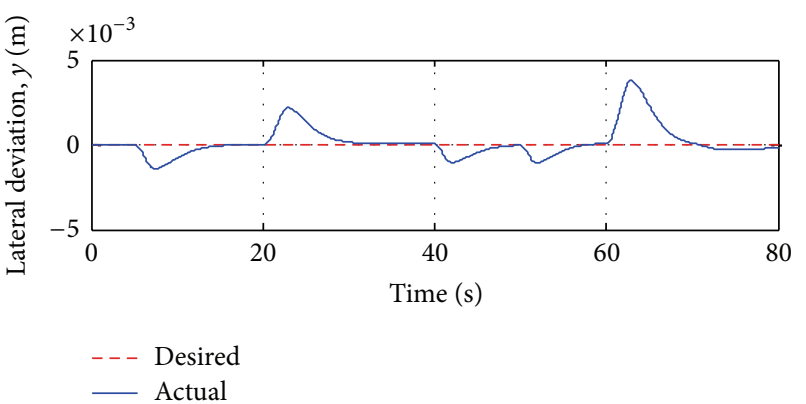

(d)

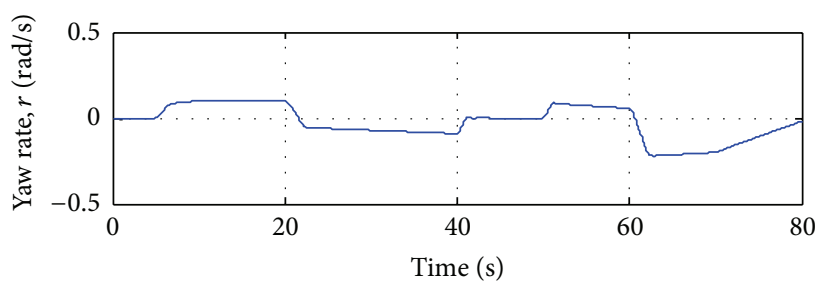

FIGURE 13: Simulation results for curved trajectory.

as $(15,12.5)$ from the shaded area in Figure 11 which satisfies the design requirements for all operating points.

\section{Simulation Study}

The simulation studies are performed to test the effectiveness of the designed robust PID controller. In the first simulation, vehicle mass, vehicle velocity, and road friction coefficient are taken as $1500 \mathrm{~kg}, 15 \mathrm{~m} / \mathrm{s}$, and 1, respectively. These parameter values correspond to the point marked with $S$ in the uncertainty box of Figure 2 .

In the first simulation of Figure 12, the vehicle tries to follow a path consisting of a straight track of $150 \mathrm{~m}$ followed by a full turn in a circle of radius $100 \mathrm{~m}$ followed by a $150 \mathrm{~m}$ straight track. The vehicle velocity is kept constant at $15 \mathrm{~m} / \mathrm{s}$ along the way. In the nonlinear vehicle model, a PI-based cruise control algorithm keeps the vehicle velocity constant. Also, the steering angle saturation is taken into consideration in the simulations. The front wheel steering angle $\delta_{f}$ is limited to 40 degrees. The simulation results given in Figure 12 show the vehicle trajectory, the lateral deviation, the vehicle velocity, and the yaw rate. It is seen that the vehicle follows the desired trajectory successfully. The vehicle velocity is kept around $15 \mathrm{~m} / \mathrm{s}$ by the cruise control algorithm and the vehicle yaw rate is at acceptable values.

In the second simulation, the vehicle tries to follow a curved path with different road curvature values. The tireroad friction coefficient alters between 1 and 0.5 to simulate different road conditions such as dry asphalt and slippery road surface. The vehicle tries to track a velocity profile which changes between 5 and $18 \mathrm{~m} / \mathrm{sec}$. The followed road curvature, the variable tire-road friction coefficient profile, the vehicle trajectory, the lateral deviation of the vehicle, the velocity profile followed, the vehicle velocity, and also the vehicle yaw rate change are shown in Figure 13. It is seen that the vehicle follows the given trajectory with very small lateral deviation and that the velocity profile is followed successfully by the PI-based cruise controller. The vehicle yaw rate is also at acceptable values.

\section{Conclusions}

A parameter space based robust PID steering controller design for automated steering was developed and tested in 
a simulation environment in this paper. A validated model of a midsized sedan was used in the design and simulations. The simulation results showed the success of this controller in path following. This study is part of a larger research effort aimed at highly automated driving. The steering controller designed here will be implemented and used in the experimental vehicle Okanom presented in this paper.

\section{Conflict of Interests}

The authors declare that there is no conflict of interests regarding the publication of this paper.

\section{Acknowledgments}

The authors would like to thank the İstanbul Okan University Transportation Technologies and Intelligent Automotive Systems Application and Research Center (UTAS) and TofaşFiat. Also, the first and the second authors would like to thank the support of TÜBİTAK (The Scientific and Technological Research Council of Turkey) National Scholarship Programme for PhD Students.

\section{References}

[1] K. L. R. Talvala, K. Kritayakirana, and J. C. Gerdes, "Pushing the limits: from lanekeeping to autonomous racing," Annual Reviews in Control, vol. 35, no. 1, pp. 137-148, 2011.

[2] L. Güvenç, İ. M. C. Uygan, K. Kahraman et al., "Cooperative adaptive cruise control implementation of team mekar at the grand cooperative driving challenge," IEEE Transactions on Intelligent Transportation Systems, vol. 13, no. 3, pp. 1062-1074, 2012.

[3] A. Broggi, P. Medici, P. Zani, A. Coati, and M. Panciroli, "Autonomous vehicles control in the VisLab intercontinental autonomous challenge," Annual Reviews in Control, vol. 36, no. 1, pp. 161-171, 2012.

[4] K. A. Ünyelioğlu, C. Hatipoğlu, and Ü. Özgüner, "Design and stability analysis of a lane following controller," IEEE Transactions on Control Systems Technology, vol. 5, no. 1, pp. 127134, 1997.

[5] A. Broggi, M. Bertozzi, A. Fascioli, C. G. LoBianco, and A. Piazzi, "The ARGO autonomous vehicle's vision and control systems," International Journal of Intelligent Control and Systems, vol. 3, no. 4, pp. 409-441, 1999.

[6] J. Ackermann, J. Guldner, W. Sienel, R. Steinhauser, and V. I. Utkin, "Linear and nonlinear controller design for robust automatic steering," IEEE Transactions on Control Systems Technology, vol. 3, no. 1, pp. 132-143, 1995.

[7] B. Aksun Güvenç and L. Güvenç, "Robust two degree-offreedom add-on controller design for automatic steering," IEEE Transactions on Control Systems Technology, vol. 10, no. 1, pp. 137-148, 2002.

[8] R. Marino, S. Scalzi, and M. Netto, "Nested PID steering control for lane keeping in autonomous vehicles," Control Engineering Practice, vol. 19, no. 12, pp. 1459-1467, 2011.

[9] J. Ackermann, P. Blue, T. Bunte et al., Robust Control: The Parameter Space Approach, Springer, London, UK, 2002.

[10] B. Aksun Güenç, L. Güvenç, and S. Karaman, "Robust yaw stability controller design and hardware-in-the-loop testing for a road vehicle," IEEE Transactions on Vehicular Technology, vol. 58, no. 2, pp. 555-571, 2009.

[11] B. Aksun Güvenç, L. Güvenç, and S. Karaman, "Robust MIMO disturbance observer analysis and design with application to active car steering," International Journal of Robust and Nonlinear Control, vol. 20, no. 8, pp. 873-891, 2010.

[12] K. Kahraman, M. T. Emirler, M. Şentürk, B. Aksun Güvenç, L. Güvenç, and B. Efendioğlu, "Estimation of vehicle yaw rate using a virtual sensor with a speed scheduled observer," in Proceedings of the IFAC Symposium on Advances in Automotive Control, pp. 632-637, Munich, Germany, July 2010.

[13] Autonomous Driving Demo at Istanbul Okan University, http:// www.youtube.com/watch? $\mathrm{v}=6 \mathrm{MQdX} 4$ _dx $4 \mathrm{U}$.

[14] Okan Autonomous Vehicle Project Presentation, http://arastirma.okan.edu.tr/sayfa/okanom-otonom-arac-projesi-tanitimi.

[15] İ. Altay, B. Aksun Güvenç, and L. Güvenç, "A simulation study of GPS/INS integration for use in ACC/CACC and HAD," in Proceedings of the 9th Asian Control Conference, İstanbul, Turkey, June 2013. 

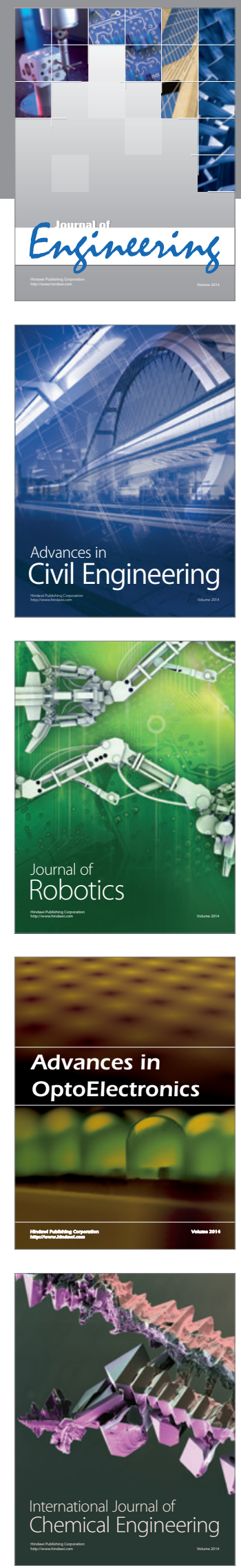

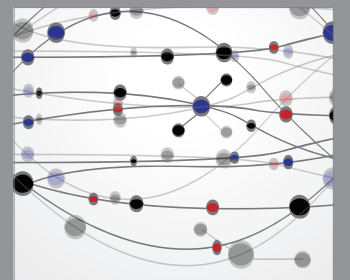

The Scientific World Journal
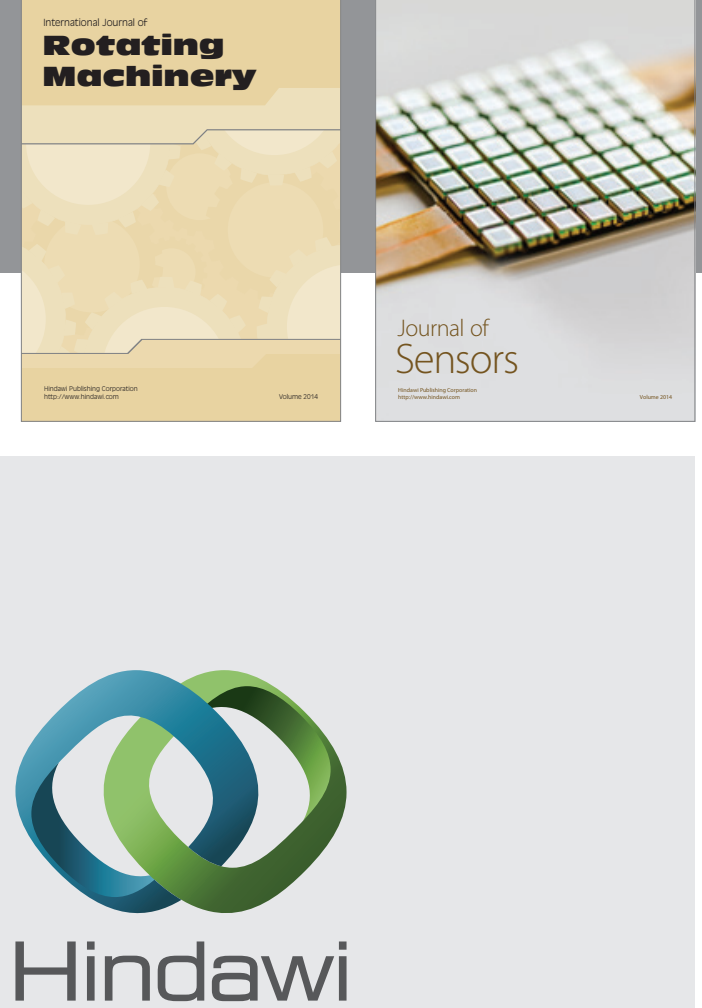

Submit your manuscripts at http://www.hindawi.com
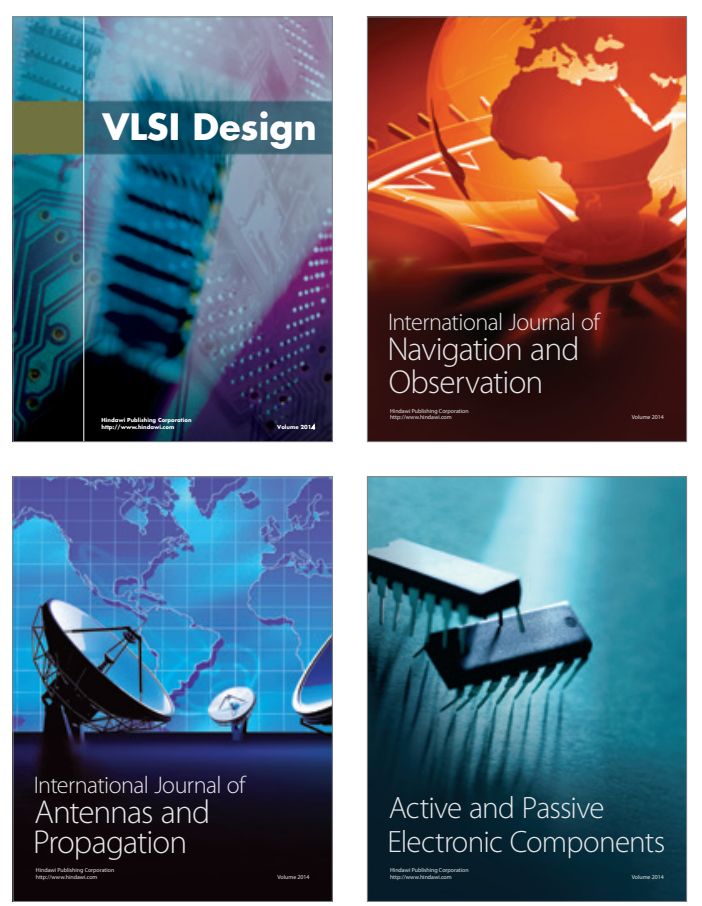
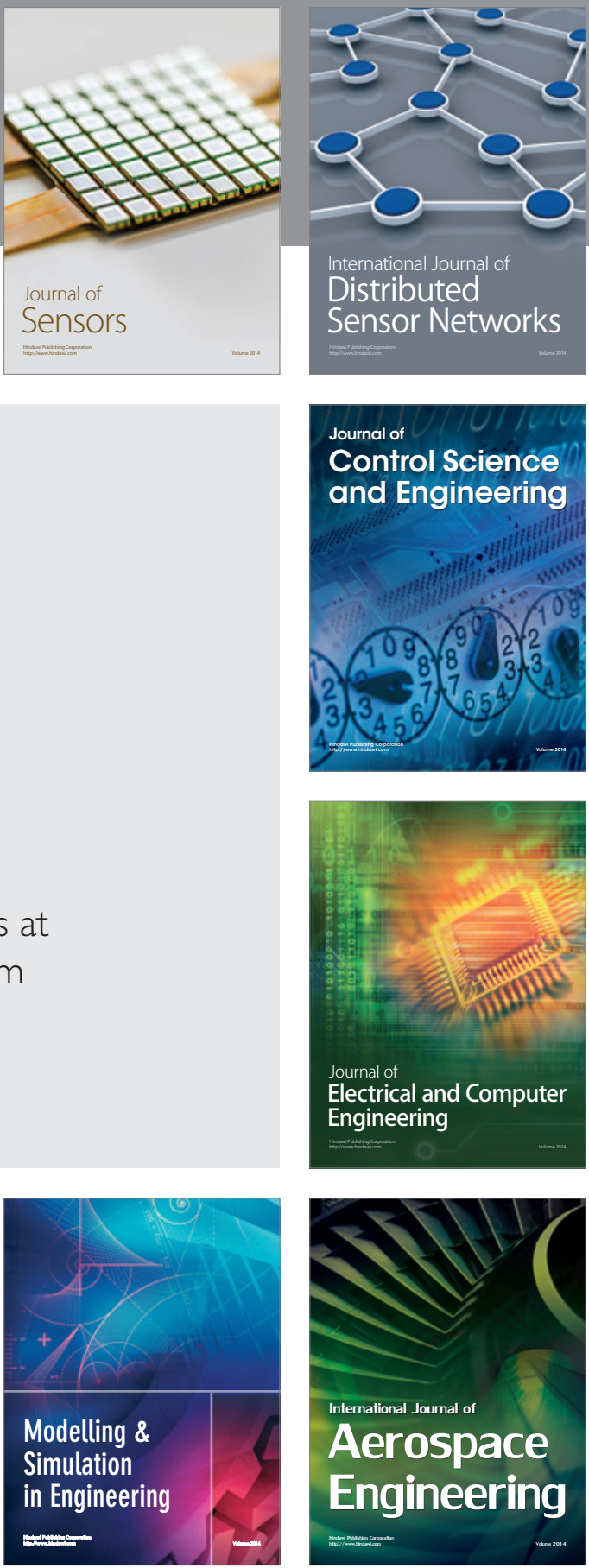

Journal of

Control Science

and Engineering
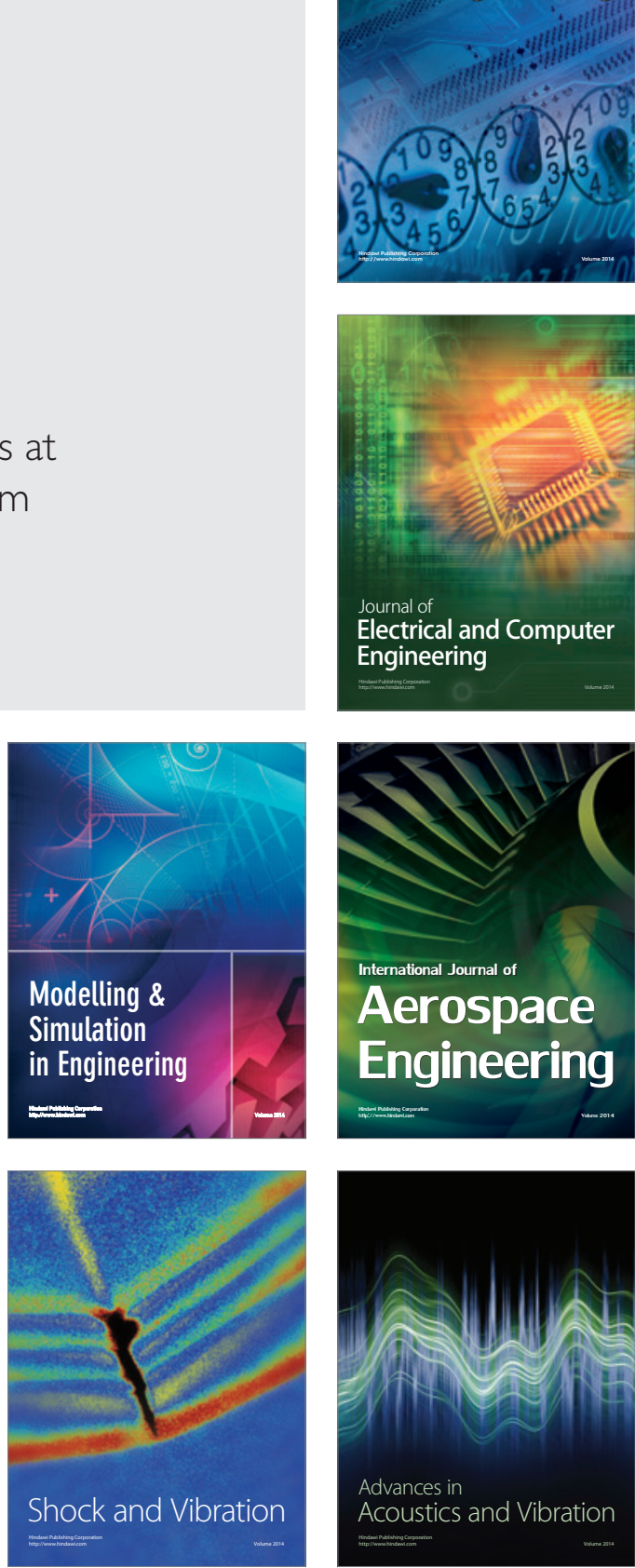